\title{
REVIEW
}

\section{A systematic review of adverse events in the placebo arm of donepezil trials: the role of cognitive impairment}

\author{
Martina Amanzio, ${ }^{1,2}$ Fabrizio Benedetti ${ }^{3,4}$ and Lene Vase ${ }^{5,6}$ \\ ${ }^{1}$ Department of Psychology, University of Turin, Turin, Italy \\ ${ }^{2}$ Neuroscience Institute of Turin (NIT), Turin, Italy \\ ${ }^{3}$ Department of Neuroscience, University of Turin Medical School, Turin, Italy \\ ${ }^{4}$ National Institute of Neuroscience (INN), Turin, Italy \\ ${ }^{5}$ Department of Psychology, Aarhus University, Aarhus, Denmark \\ ${ }^{6}$ Danish Pain Research Center, Aarhus University Hospital, Noerrebrogade 44, DK- 8000 Aarhus C, Denmark
}

Background: In randomized clinical trials, adverse events (AEs) are reported for the drug under evaluation and compared with the placebo group. Patients who receive placebo treatment report a high frequency of AEs, but little is understood about the nature of these. No study has yet analyzed the level of cognitive impairment as a crucial aspect for the AEs reported by patients.

Methods: The rates of AEs reported by patients with mild cognitive impairment (MCI) and Alzheimer's disease $(\mathrm{AD})$ in the placebo arms of donepezil trials were compared using a systematic review approach. PubMed was searched with the terms "MCI and donepezil" as well as "AD and donepezil" from January 1989 to December 2010. Nineteen studies fulfilled the selection criteria ( $3 \mathrm{MCI}, \mathrm{n}=783 ; 16 \mathrm{AD}, \mathrm{n}=2,059$ ).

Results: An overall comparison of 81 categories of AEs in the placebo arm of MCI versus AD trials showed that patients in $\mathrm{AD}$ trials experienced a significantly higher number of AEs than patients in MCI trials $(\mathrm{p}<$ $0.001)$.

Conclusions: This is the first study showing that $\mathrm{AD}$ patients may be at a greater risk of developing AEs than MCI patients. This may be related to a greater presence of somatic comorbidity predisposing them to express emotional distress as physical symptoms and/or to $\mathrm{AD}$ patients being frailer and therefore more susceptible to AEs. The phenomena we observed may be interpreted in terms of the "nocebo effect".

Key words: Alzheimer's disease, mild cognitive impairment, randomized controlled trials, nocebo

\section{Introduction}

The adverse events (AEs) that may occur in pharmacological treatments of patients with cognitive deterioration need careful investigation in order to minimize the risk and maximize the benefit of drugs. The safety profile has shown that the risks associated with cholinesterase inhibitors (ChEIs) are not negligible (Raschetti et al., 2007). The most common AEs of ChEIs are cholinergic symptoms such as nausea, vomiting, diarrhea, muscle cramps, dizziness, fatigue, and anorexia (Standaert and Young, 1996). The data obtained by Doody et al. (2010) suggest that patients with Alzheimer's

Correspondence should be addressed to: Dr. Martina Amanzio, Department of Psychology, University of Turin, Via Verdi 10, 10123, Turin, Italy. Phone: +39-11-6702468; Fax: +39-11-6702061. Email: martina.amanzio@unito.it. Received 15 Jun 2010; revision requested 2 Sep 2011; revised version received 7 Oct 2010; accepted 20 Oct 2011. First published online 4 January 2012. disease $(\mathrm{AD})$ tolerate donepezil better than patients with mild cognitive impairment (MCI); however, no study has yet investigated published randomized clinical trials (RCTs) of donepezil that have reported rates of AEs in the placebo groups of MCI and $\mathrm{AD}$ patients.

We recently demonstrated that informing subjects about the AEs that they may possibly experience could have a significant impact on their actual experience and report of AEs (Amanzio et al., 2009). This phenomenon can be observed not only in the active group but also in the placebo group of RCTs (for a review, see Amanzio, 2011). Although the underlying mechanisms of this phenomenon remain unclear, the nocebo effect may offer an important theoretical framework to interpret the obtained data (Amanzio et al., 2009; Rief et al., 2009). A nocebo effect is defined as the negative outcome following administration 
of an inert substance along with suggestions of negative modifications of symptoms or sensations (Benedetti and Amanzio, 1997; Harrington, 1997; Benedetti et al., 2007). In particular, the knowledge about taking medication or the anxiety about illness course may cause patients to monitor side effects in more detail, resulting in an amplified perception of physical symptoms. Interestingly, somatization, a phenomenon whereby the patient expresses emotional distress in the form of physical symptoms, has previously been associated with nocebo symptoms and with the occurrence of AEs in relation to active drugs (Wolf and Pinsky, 1954; Andrykowski and Redd, 1987; Davis et al., 1995).

Since behavioral and psychological symptoms of dementia may be worsened or caused by somatic comorbidity, such as anxiety, depression, cardiovascular disease, infections, delirium, falls, incontinence, and anorexia (Doraiswamy et al., 2002; Waldemar et al., 2007), and since there is a strong association between somatic comorbidity and cognitive status in patients with dementia (see Doraiswamy et al., 2002; Waldemar et al., 2007), it will be interesting to analyze the presence of AEs in patients with different levels of cognitive impairment.

We therefore conducted an analysis of published RCTs involving $\mathrm{MCI}$ and $\mathrm{AD}$ patients receiving donepezil and reporting AEs in the placebo arms of the trial. We hypothesized that the information about AEs would lead patients to report symptoms in the corresponding placebo groups. We also hypothesized that the level of AEs may differ across patients with varying degrees of cognitive impairment so that MCI and $\mathrm{AD}$ patients would report a significantly different number of AEs.

\section{Methods}

\section{Search procedure}

We conducted a search in the Medline database (PubMed http://pubmed.gov/) with the following limits: Humans, Randomized Clinical Trial, English, and dates from 1 January 1989 to 31 December 2010. The search terms were "Mild Cognitive Impairment and donepezil" and "Alzheimer's disease and donepezil." The final version of the search was conducted on 17 January 2011 .

\section{Inclusion and exclusion criteria}

Trials were selected for inclusion or exclusion according to the following criteria: (1) only placebocontrolled RCTs comparing donepezil versus placebo in MCI or AD patients were considered;
(2) only trials that reported AEs in the placebo condition were included; (3) we excluded trials with insufficient reporting of AEs (i.e. either no reports of side effects at all or studies that reported "treatment-related AEs" without specifying these further); (4) crossover studies were excluded from the analysis (if placebo is given as the first treatment, one is measuring the effects of suggestion only, whereas if placebo is given as a second treatment, one is measuring the effects of both suggestion and conditioning); (5) trials comparing two classes of active drugs or multiple treatments in the same trial were excluded; and (6) duplicate publications were also excluded.

\section{Recording and grouping of AEs}

First, AEs from each single trial were recorded. This yielded a total of 90 categories of AEs. Some of these AEs were either semantically similar (e.g. fever and pyrexia) or meaningfully related (e.g. nausea and vomiting) and were therefore grouped together. Thus, a total of 81 categories of AEs were identified.

Second, for some of the categories of AEs the presence of specific AEs was only reported in two or less trials. These AEs were therefore removed from the analysis. This procedure reduced the number of AEs to 21 of different categories.

Third, some AEs were only reported in trials involving MCI patients and others only in trials involving $\mathrm{AD}$ patients. It was therefore possible to directly compare the number of AEs in MCI versus $\mathrm{AD}$ patients in relation to the following seven categories of AEs: depression, diarrhea, dizziness, headache, insomnia, muscle cramps, and nausea/vomiting (see Figure 1).

In most of the studies, AEs were reported on the basis of a single measure. However, in a few studies AEs were divided into serious AEs and nonserious AEs (e.g. Homma et al., 2000; Mohs et al., 2001; Windblad et al., 2001). In such cases, serious and non-serious AEs belonging to the same category were grouped together to make the recording of AEs consistent across studies.

Importantly, most of the studies only described AEs that were reported by at least $5 \%$ of the patients in the donepezil and placebo groups. However, a few studies also included AEs reported by less than $5 \%$ of the patients (e.g. Rogers et al., 1998a; 1998b; Homma et al., 2000; Johannesen et al., 2006). In these studies the authors did not separately list "AEs reported by less than $5 \%$ of the patients" versus "AEs reported by more than $5 \%$ of the patients," and hence all AEs reported in the studies were included in the systematic review. 
I Total number of AEs

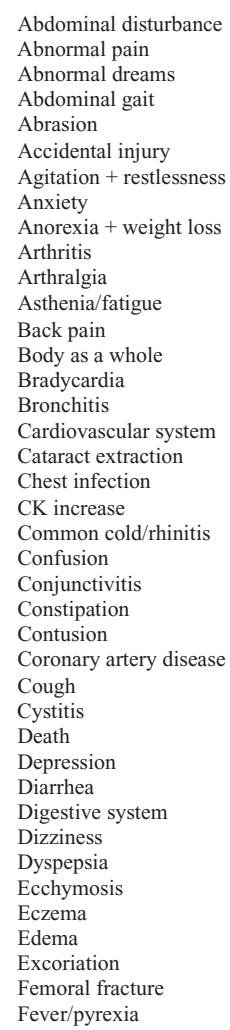

\begin{tabular}{|c|c|}
\hline 41. & Flushing \\
\hline 42. & Fracture \\
\hline 43. & Gastroenteritis \\
\hline 44. & Gastrointestinal bleeding \\
\hline 45. & Headache \\
\hline 46. & Hemorrhage \\
\hline 47. & Hostility \\
\hline 48. & Infection \\
\hline 49. & Injury \\
\hline 50. & Insomnia \\
\hline 51. & Leg cramps \\
\hline 52. & Loose stool \\
\hline 53. & Loss of appetite \\
\hline 54. & Muscle cramps \\
\hline 55. & Muscoloskeletal system \\
\hline 56. & Myasthenia \\
\hline 57. & Myocardial infection \\
\hline 58. & Nasal congestion \\
\hline 59. & Nasophyrangitis \\
\hline 60 . & Nausea + vomiting \\
\hline 61. & Nervous system \\
\hline 62. & Pain \\
\hline 63. & Pneumonia \\
\hline 64. & Postural hypotension \\
\hline 65. & Procedure (medical + surgical + healt \\
\hline 66. & Rash \\
\hline 67. & Respiratory system \\
\hline 68. & Respiratory tract infection \\
\hline 69. & Seizure \\
\hline 70 . & Sinus bradycardia \\
\hline 71. & Skin ulcer \\
\hline 72. & Somnolence \\
\hline 73. & Stomach upset \\
\hline 74. & Stroke \\
\hline 75. & Stupor \\
\hline 76. & Syncope \\
\hline 77. & Tremor \\
\hline 78. & Upper respiratory tract inflammation \\
\hline 79. & Urinary tract infection \\
\hline 80. & Urogenital system \\
\hline 81. & Vertigo \\
\hline
\end{tabular}

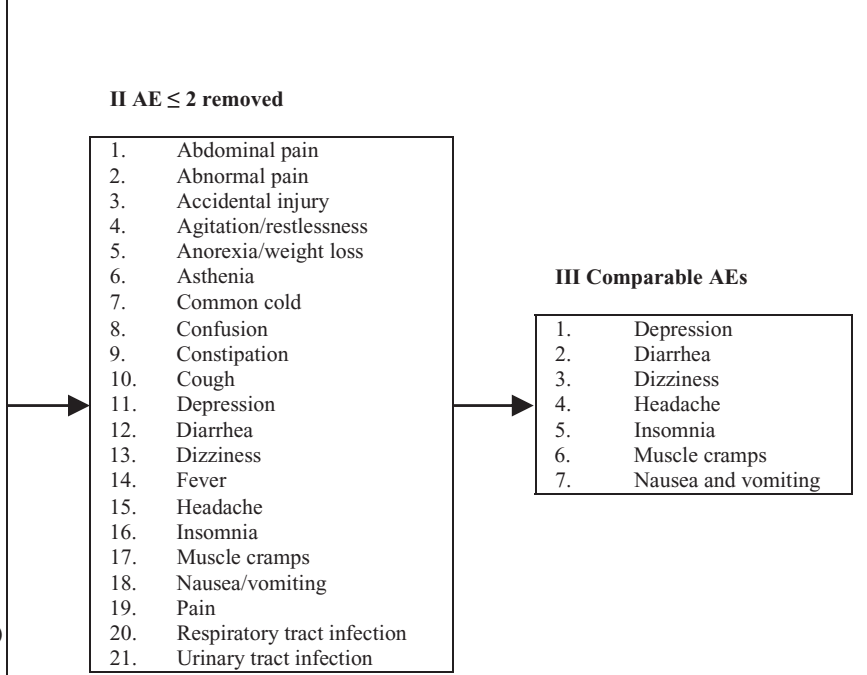

Figure 1. Flow chart for categories of AEs. I: The total number of AEs identified across all MCI and AD studies. II: The remaining AEs when all the AEs that only occurred in up to a maximum of two studies were removed. III: The final sample of AEs with a sufficient number of trials in both $\mathrm{MCl}$ and $\mathrm{AD}$ samples to allow for a statistical comparison.

\section{Data abstraction and validity assessment}

Each trial was coded using a structured coding scheme (the full version can be requested from the corresponding author), including information on patients, treatment, and methodology. For each trial, we noted the year of publication, duration of the trial, quality of the trial evaluated by means of the Jadad scores (Jadad et al., 1996; these scores consider the description and sequence of randomization, the double-blind procedure, its appropriateness, and the description of withdrawals: range 0-5), the assessment strategy (structured versus spontaneous reports of AEs), age, gender, race, Mini Mental State Examination (MMSE; Folstein et al., 1975), general withdrawal, and withdrawal due to AEs.

Furthermore, we noted the number of patients who reported AEs in the placebo group. We then summed the counts of separate studies in order to obtain the cumulative number of patients reporting the $\mathrm{AE}$ to combine data from individual studies. We considered no report of AEs in a trial as absence of AEs in the placebo group of that trial. In other words, in this case the trial was equated to a trial explicitly reporting absence of the symptom (0 value).
Adverse events, quality of the study, and assessment strategy were recorded independently for each study by two different researchers (MA and LV).

\section{Data analysis}

SPSS 19.0 was used and $\mathrm{p}<0.05$ was considered significant. The results were expressed as mean values and standard deviations.

Mean inter-rater reliability with respect to recording of AEs for each individual trial was $\kappa=0.89$. Mean inter-rater reliability for trial quality assessment (in terms of the Jadad scores) was $\kappa=0.78$ and the assessment strategy in terms of structured versus spontaneous recording of AEs had a mean inter-rater reliability of $\kappa=0.81$.

First, in order to test whether trials involving AD and MCI patients were comparable, we compared these two types of trials with respect to year of publication, duration of study, quality of study (Jadad score), assessment strategy (structured vs. spontaneous recording of AEs), age, gender, race, MMSE scores, general withdrawal, and withdrawal due to AEs. As both age and race were calculated as the ratio of females and Caucasians within each trial, respectively, it was possible to calculate all of the above variables via independent $t$-test. 
Table 1. Characteristics of the placebo groups in the mild cognitive impairment $(\mathrm{MCl})$ and Alzheimer's disease (AD) trials. The table illustrates that $\mathrm{MCl}$ and $\mathrm{AD}$ studies are comparable.

\begin{tabular}{|c|c|c|c|c|c|c|c|}
\hline & \multirow[b]{2}{*}{ VALID N } & \multicolumn{3}{|c|}{ MCI } & \multicolumn{3}{|c|}{$\mathrm{AD}$} \\
\hline & & MEAN & SD & RANGE & MEAN & SD & RANGE \\
\hline Year of publication & 19 & 2006 & 2.646 & 2004-2009 & 2002.44 & 3.829 & 1999-2008 \\
\hline Duration of trial (weeks) & 19 & 36 & 12.000 & $24-48$ & 24.75 & 12.261 & $12-54$ \\
\hline Quality of the trial (Jadad $\left.{ }^{a}\right)$ & 19 & 4 & 1 & $3-5$ & 4.38 & 0.719 & $3-5$ \\
\hline Age (years) & 19 & 71.67 & 1.528 & $70-73$ & 75.63 & 5.402 & $70-86$ \\
\hline Gender (percentage of females) & 19 & 43.90 & 2.45 & $42.34-46.72$ & 66.09 & 10.09 & $52.50-87.02$ \\
\hline Race (percentage of Caucasians) & 11 & 88.99 & 5.25 & $85.27-92.70$ & 102.38 & 25.93 & $76.05-173.21$ \\
\hline MMSE & 19 & 27.38 & 0.289 & $27.35-27.40$ & 15.496 & 5.479 & $6.20-24.30$ \\
\hline General withdrawal & 19 & 67.67 & 45.523 & $23-114$ & 24.44 & 17.270 & $1-56$ \\
\hline Withdrawal due to AEs & 19 & 21.00 & 15.556 & $10-32$ & 10.31 & 7.878 & $0-27$ \\
\hline
\end{tabular}

a Jadad evaluation of trial quality.

AEs = adverse events; MMSE = Mini-Mental State Examination (lower scores indicate more severe cognitive impairment); SD = standard deviation.

Second, in order to test whether there was an association between the level of cognitive impairment and the presence-absence of AEs in placebo groups, Fisher's exact tests were performed to analyze whether there were significant differences in the frequency of AEs in the placebo arm of trials involving $\mathrm{AD}$ and $\mathrm{MCI}$ patients. In particular, (1) the overall level of AEs was compared for MCI and $\mathrm{AD}$ patients for all 81 categories of AEs; (2) the specific levels of AEs in MCI versus $\mathrm{AD}$ trials were compared in each of the seven categories of AEs where there were sufficient reports of symptoms in both MCI and AD trials to allow for a direct comparison (depression, diarrhea, dizziness, headache, insomnia, muscle cramps, and nausea/vomiting).

Finally, in order to test the variation in the cognitive deterioration, homogeneity analyses were conducted on the MMSE scores (http://www.childrensmercey.org/stats/model/metaanalysis.asp). The data could not be meaningfully analyzed for publication bias and therefore this analysis was omitted.

\section{Results}

One hundred and forty-nine trials were identified. Eighty-three trials (4 MCI and $79 \mathrm{AD}$ ) were excluded because they were not placebo-controlled RCTs comparing donepezil versus placebo in $\mathrm{AD}$ or MCI patients. A further 30 trials ( 2 on MCI and 28 on AD) were excluded because they did not fulfill one or more of the subsequent selection criteria (see Method section). Of the remaining 36 articles, 17 were excluded (13 on MCI and 4 on $\mathrm{AD}$ ) as they were double publications or publications involving the same initial group of patients. This left a total of 19 trials, which were included in the study (see Figure 2). Three trials involved MCI patients $(\mathrm{n}=783)$ and 16 trials involved AD patients $(n=2,059)$. The excluded trials are listed in the Appendix A1 along with the reason for their exclusion (see the supplementary online material attached to the electronic version of this paper at http://www.journals.cambridge.org/ipg).

\section{Comparison of MCI and AD patients}

The characteristics of the placebo arms of the MCI and $\mathrm{AD}$ trials included in the analysis are shown in Table 1 . There were no significant differences between $\mathrm{MCI}$ and $\mathrm{AD}$ trials with respect to the year of publication $(\mathrm{p}=0.145)$, duration of the trial $(\mathrm{p}=$ $0.162)$, quality of the trial $(\mathrm{p}=0.442)$, age $(\mathrm{p}=$ $0.265)$, race $(\mathrm{p}=0.499)$, general withdrawal $(\mathrm{p}=$ $0.240)$, and withdrawal due to AEs $(p=0.130)$. As far as the assessment strategy is concerned, all three of the MCI studies and 15 out of $16 \mathrm{AD}$ studies were unstructured $(p=0.842)$. However, the ratio of females was significantly higher in the $\mathrm{AD}$ studies as compared with the MCI studies $(p=0.002)$. Furthermore, as expected, trials involving MCI and AD patients had significantly different MMSE scores $(\mathrm{p}<0.001)$, showing that $\mathrm{AD}$ patients had a higher degree of cognitive impairment.

\section{Overall report of AEs in $\mathrm{MCI}$ versus $\mathrm{AD}$ patients}

The number and percentage of AEs across the placebo groups of MCI and AD trials can be seen in Table 2. When looking at the total number of AEs across all 81 categories, a significantly higher number of symptoms were reported in trials involving $\mathrm{AD}$ patients as compared with trials 


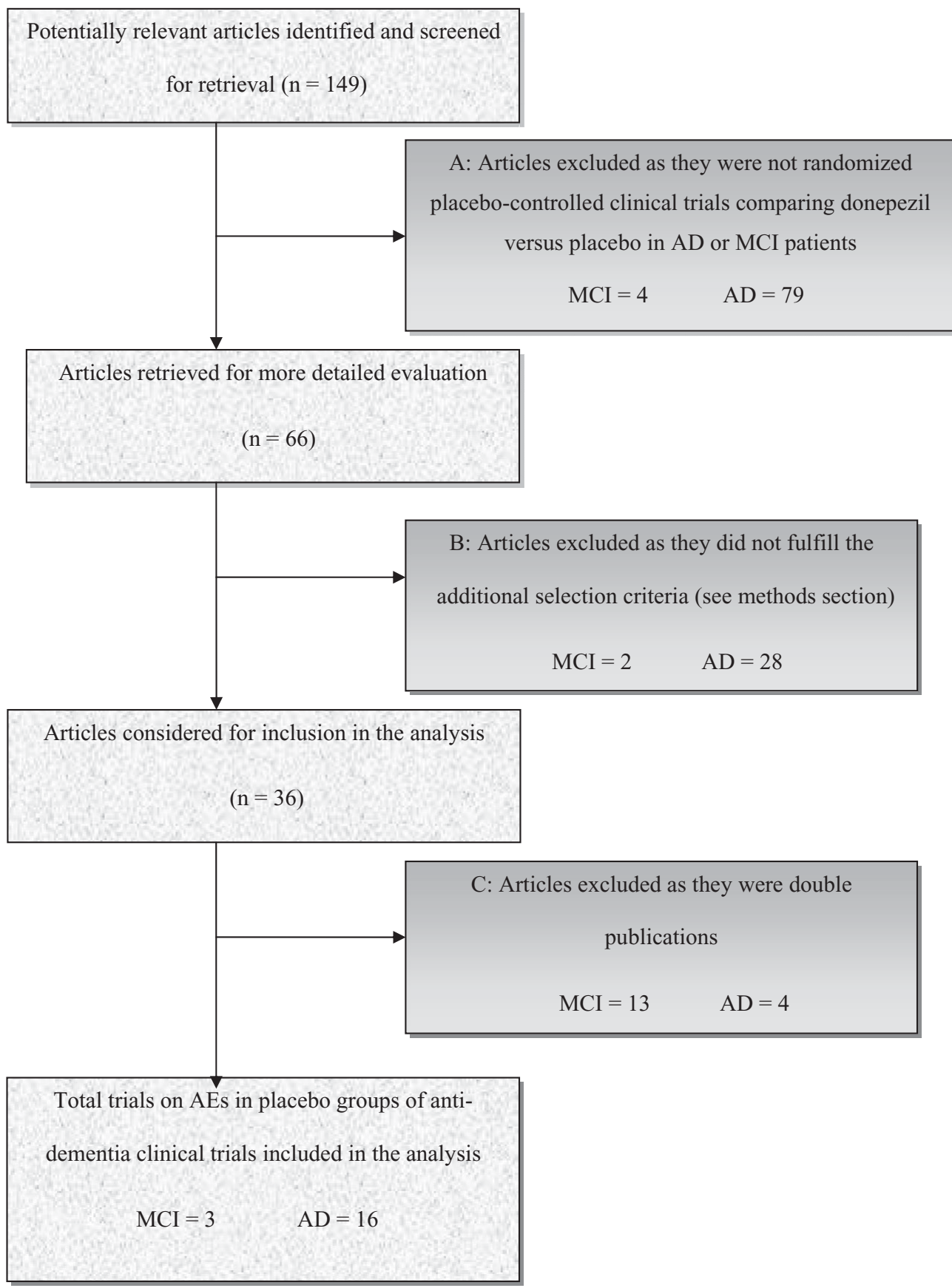

Figure 2. Flow chart for inclusion of trials. Excluded trials are shown in Appendix I (see supplementary online material attached to the electronic version of this paper at www.journals.cambridge.org/jid_IPG).

involving MCI patients $(\mathrm{p}<0.001)$. As indicated by the Cohen's $d$ of -0.954 , there was a notable difference in the total number of AEs reported in $\mathrm{AD}$ and MCI trials (Table 2 and Figure 3).

\section{Direct comparison of seven types of AEs in MCI versus AD patients}

When looking at the seven categories of AEs where a direct comparison between $\mathrm{AD}$ and MCI trials was possible, $\mathrm{AD}$ patients experienced depression $(\mathrm{p}=0.016)$, dizziness $(\mathrm{p}=0.007)$, headache $(\mathrm{p}=0.030)$, and nausea/vomiting $(\mathrm{p}=0.036)$ significantly more frequently than MCI patients. We found no significant difference between MCI and $\mathrm{AD}$ trials with respect to the frequency of the remaining AEs (for diarrhea $\mathrm{p}=0.425$; for insomnia $\mathrm{p}=0.613$; and for muscle cramps $\mathrm{p}=0.806$ ) (see Table 2 and Figure 3). Only dizziness survived the Bonferroni correction of the alpha value $(0.05 / 7=$ $\leq 0.007$ ), which is most likely because for some of the AEs there were only few reports in each category 
Table 2. Number of adverse events in placebo groups of the mild cognitive impairment (MCI) and Alzheimer's disease $(A D)$ trials. The table shows that trials with $A D$ patients report a significantly higher number of $A E s$ than trials with $\mathrm{MCl}$ patients.

\begin{tabular}{|c|c|c|c|c|c|c|c|c|c|c|c|c|}
\hline \multirow[b]{2}{*}{ SYMPTOMS } & \multicolumn{5}{|c|}{ MCI } & \multicolumn{5}{|c|}{$\mathrm{AD}$} & \multirow{2}{*}{$\begin{array}{c}\text { MCI VS. } \\
\text { AD } \\
\text { COHEN'S } d\end{array}$} & \multirow{2}{*}{$\begin{array}{l}\text { MCI VS. } \\
\text { AD } \\
\text { P-VALUE }\end{array}$} \\
\hline & $\mathrm{N}$ & $n$ & $\%$ & $\begin{array}{c}\text { LOWER } \\
\text { CI }\end{array}$ & $\begin{array}{c}\text { UPPER } \\
\text { CI }\end{array}$ & $\mathrm{N}$ & $n$ & $\%$ & $\begin{array}{c}\text { LOWER } \\
\text { CI }\end{array}$ & $\begin{array}{c}\text { UPPER } \\
\text { CI }\end{array}$ & & \\
\hline Total AEs & 783 & 180 & 22.989 & 20.041 & 25.936 & 2,059 & 1,373 & 66.683 & 64.647 & 68.719 & -0.954 & 0.0000 \\
\hline Depression & 137 & 1 & 0.730 & -0.696 & 2.155 & 290 & 16 & 5.517 & 2.889 & 8.145 & -0.246 & 0.016 \\
\hline Diarrhea & 783 & 40 & 5.109 & 3.566 & 6.651 & 2,059 & 91 & 4.420 & 3.532 & 5.307 & 0.033 & 0.425 \\
\hline Dizziness & 259 & 4 & 1.544 & 0.0426 & 3.046 & 1,081 & 57 & 5.273 & 3.941 & 6.605 & -0.179 & 0.007 \\
\hline Headache & 387 & 12 & 3.101 & 1.374 & 4.828 & 975 & 59 & 6.051 & 4.555 & 7.548 & -0.133 & 0.030 \\
\hline Insomnia & 783 & 29 & 3.704 & 2.381 & 5.027 & 845 & 36 & 4.260 & 2.899 & 5.622 & -0.028 & 0.613 \\
\hline Muscle cramps & 646 & 12 & 1.858 & 0.816 & 2.899 & 315 & 7 & 2.222 & 0.594 & 38.501 & -0.026 & 0.806 \\
\hline Nausea/vomiting & 782 & 36 & 4.604 & 3.135 & 6.072 & 2,059 & 139 & 6.751 & 5.667 & 7.835 & -0.089 & 0.036 \\
\hline
\end{tabular}

$\mathrm{N}=$ total sample size, $n=$ number of patients reporting AEs.

Values are given as percentages and $95 \%$ confidence intervals (CI) are indicated (lower: lower value of the interval, upper: upper value of the interval).

Cohen's $d$ : a negative value indicates that AEs are reported more frequently in AD trials as compared with MCI trials.

p-value (2-sided) following the Fisher's exact test.

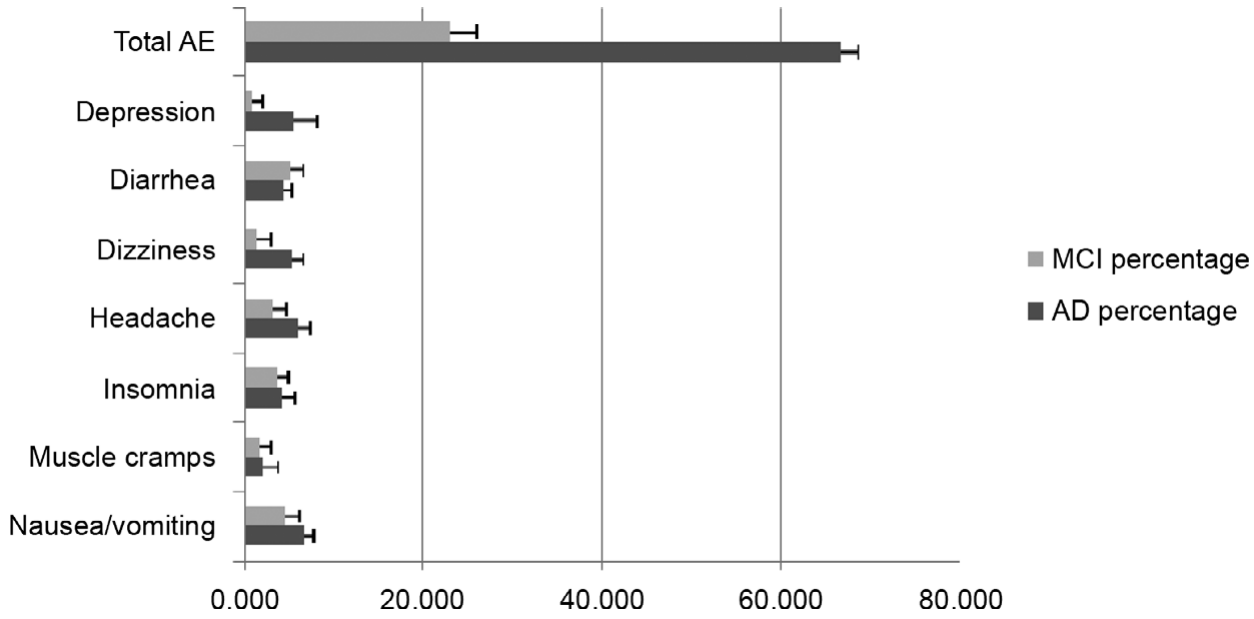

Figure 3. Bar chart plots of the percentage of $A E s$ in the placebo arms of $\mathrm{MCl}$ and $A D$ trials. The error bars (thin black line) refer to the confidence intervals of each percentage. The figure illustrates that all but one category of $A E$ trials with AD patients report more AEs than trials with $\mathrm{MCl}$ patients.

(e.g. depression 136 vs. 1 and 274 vs. 16). However, as can be seen from the Cohen's $d$ in Table 2, with the exception of the symptom of diarrhea, all comparisons of AEs indicate that patients in $\mathrm{AD}$ trials report a higher number of AEs than patients in MCI trials, and the overall result strongly supports that patients in $\mathrm{AD}$ trials actually experience AEs to a significantly higher extent than MCI patients. Thus, overall, all the results go in the same direction and for the total sample this is strongly significant.

\section{Homogeneity analyses}

The homogeneity analyses of the MMSE scores showed $\mathrm{I}^{2}=0$ for the MCI trials and $\mathrm{I}^{2}=$
94.6 for AD trials. This indicates that the MMSE scores in the MCI trials were highly homogeneous, whereas the MMSE scores in the AD trials were not homogeneous. This was an obvious result corresponding to a higher range of cognitive impairment in $\mathrm{AD}$ patients, from a mild to a very impaired cognitive state.

\section{Discussion}

\section{Adverse events in the placebo arm of donepezil RCT trials}

These results show that $\mathrm{AD}$ patients in the placebo arm of donepezil RCTs experienced $\mathrm{AEs}$ and that these AEs are similar to the 
symptoms observed in the active group. Indeed, active treatment with donepezil induces cholinergic AEs such as nausea, vomiting, diarrhea, muscle cramps, and dizziness (Waldemar et al., 2007). Interestingly, $\mathrm{AD}$ patients reported a significantly higher number of AEs than MCI patients in the placebo arm of donepezil clinical trials. While the overall comparisons revealed this profound difference, the specific comparisons indicated that the differences in reported AEs might especially relate to depression, dizziness, headache, and nausea/vomiting. Our study represents the first analysis demonstrating how different levels of cognitive impairment influence the level of AEs considering $\mathrm{MCI}$ and $\mathrm{AD}$ patients in the placebo arm of RCTs.

The results we obtained may be interpreted in the direction of the nocebo effect as previously demonstrated by other systematic reviews of this type on migraineurs and psychiatric patients (Amanzio et al., 2009; Rief et al., 2009). In particular, the nocebo effect is related to somatization whereby the patient expresses emotional distress as physical symptoms. Clinicians have noted that the side effects reported by highly anxious patients are often the somatic concomitants of anxiety itself, such as tachycardia, dyspnea, or sweating (Ferguson, 1993), possibly because anxious people are monitoring their body to a higher extent and amplifying the symptoms. A tendency toward somatization, symptom amplification, and a heightened awareness of bodily sensation have also been associated with non-specific AEs to active drugs as well as with nocebo effects (Wolf and Pinsky, 1954; Andrykowski and Redd, 1987). Both of these may be crucial in the patients we analyzed in the included studies.

Although the majority of the studies we considered here did not include a systematic assessment of somatization and mood changes previous studies have shown that in patients with dementia of Alzheimer's type, there is a strong association between cognitive status and somatic comorbidity (Doraiswamy et al., 2002).

Another possible alternative explanation of the results obtained is that people with more severe $\mathrm{AD}$ might be frailer and therefore more susceptible to and suffer from more AEs. It is important to underline that nowadays frailty is recognized as a condition or phenotype that is predictive of adverse health outcomes (Fried et al., 2001; McLean and Le Couteur, 2004; Walston et al., 2006; Hilmer et al., 2007). Interestingly, the frailty "syndrome" has been variously described in terms of an excessive reduction of lean body mass and poor endurance associated with a perception of exhaustion and fatigue, high susceptibility to disease and AEs of analgesics, and an increased vulnerability to stressors (Fried et al., 2001; Cepeda et al., 2003; Walston et al., 2006).

Further studies will be fundamental to disentangle somatization from frailty and define these as separate or concomitant phenomena.

\section{Limitations of the study}

A possible limitation of the current study is that although the data are based on a total of 2,842 patients and 81 categories of AEs, it was possible to directly compare the AEs for seven categories only. Even within these seven categories of AEs there were sometimes few observations and this part of the comparison did not therefore yield strong results. This may be due in part to the fact that it was only possible to identify three studies involving MCI patients and therefore many AEs were neither reported in AD nor in MCI trials or they were reported only with a small frequency. Nevertheless, the total number of 81 categories of AEs strongly shows that trials with $\mathrm{AD}$ patients report a higher number of AEs than trials with MCI patients. Furthermore, six out of seven subcategories of AEs also show that trials with $\mathrm{AD}$ patients report a higher number of AEs than trials with MCI patients. It will be important to test these findings further as more studies emerge.

It is also important to be aware that the relationship between the level of cognitive impairment and the report of AEs deals with clinical trials and not with individual patients. In the MCI trials there was a high homogeneity in the level of cognitive functioning as indicated by the homogeneity analyses of MMSE scores. However, in the AD trials there was a high range in MMSE scores from a mild to a severe, compromised cognitive state. In the future it would be interesting to test in prospective RCTs the extent to which the individual MMSE score would be related to the presence/absence of AEs by also considering more complex neuropsychological tests and psychiatric scales, especially in early AD patients. This would help to clarify the relationship between the level of cognitive impairment and the presence of greater psychological distress predisposing people to report non-specific AEs to a greater extent.

Furthermore, in the current systematic review, all but one of the trials used an unstructured strategy for assessing AEs. However, it should be noted that the use of unstructured assessment of AEs was similar in the MCI and AD trials of the current systematic review, so the assessment strategy is unlikely to explain the difference in reporting of AEs demonstrated here. It is also important to note that 
in order for a study to qualify as using a structured strategy for assessing AEs, it would have to list a specific validated instrument for reporting AEs. Most studies did not list such an instrument but simply listed all the AEs that the patients reported. Thus, although the studies used an unstructured strategy, they did use a very similar strategy for recording AEs. Nevertheless, in future studies it would be interesting to employ more objective methods to assess AEs (Amanzio, 2011) in order to directly test the extent to which the reporting of AEs was due to cognitive dysfunctions or to unstructured reporting of AEs.

Finally, as indicated in Table 2, the rate of withdrawal due to AEs and the general rate of withdrawal were higher in MCI trials than AD trials. However, as shown in the Results section these numbers were not significantly different between the two groups and it is therefore unlikely that they would change the strong overall findings of a difference in AEs between MCI and $\mathrm{AD}$ patients.

\section{Implications of the study}

Our results suggest that $\mathrm{AD}$ patients are at greater risk of developing non-specific side effects than people with MCI. In these patients, the AEs that we observed are likely to be related to a higher presence of psychosomatic comorbidity predisposing people to express emotional distress as physical symptoms. Our study has important clinical implications, as the phenomenon we observed can involve diagnostic, nosological, and prognostic factors that directly affect treatment adherence and the course of disease. The occurrence of AEs is often related to poor clinical outcomes and impaired psychosocial functioning. These patients increase the burden on caregivers and thus require additional assistance, thereby increasing the costs of their care. Although this is a common phenomenon, it has rarely been studied and is poorly understood.

These findings also have implications for the design and interpretation of clinical trials. Our systematic review emphasizes the importance of developing standardized procedures for collecting information about AEs in double-blind placebocontrolled RCTs of the efficacy of anti-dementia drugs (see the recent review by Amanzio, 2011 for a need to create a new procedure in RCTs). Future prospective studies on $\mathrm{AD}$ patients would benefit from selecting a more homogeneous population in terms of cognitive as well as behavioral features. Indeed, considering the results that we obtained, direct intervention by the caregiver in the case of more cognitively compromised patients could have been a confounding variable. However, none of the selected articles mentioned that caregivers were reporting AEs.

Randomized clinical trials on $\mathrm{AD}$ patients should include subjects with an MMSE score ranging from 19 to 24 , corresponding to a mild stage of cognitive impairment, since it is unclear how the same methods used to assess AEs in AD studies could be entirely appropriate for patients with very extensive differences in MMSE scores. In this case, impaired language and comprehension skills could invalidate any assessment of AEs. The homogeneity of patients could also be considered in terms of the Global Deterioration Scale (i.e. they should have a score of 3 attesting to mild cognitive impairment), and in terms of clinical variables such as drug assumption and duration of illness (see Amanzio et al., 2011 for further details). Furthermore, it would be helpful to collect AEs in a structured manner and to assess mood changes and somatic comorbidity in patients with dementia not only at the time of the diagnosis but throughout the course of the disease.

When conducting an RCT of efficacy of antidementia drugs, a heightened index of suspicion is called for when the patient's symptoms are vague, ambiguous, or prevalent in daily life; when the patient has a history of negative AEs to many different classes of drugs; and when the patient is exceptionally anxious about, or even seems to expect, difficulties with the medication. Base rates of symptoms should be collected before the patient is recruited to take part in an RCT. Recently, interesting results have been found for advanced cancer patients reporting nocebo effects. Patients with, for instance, insomnia at baseline were more likely to report more nocebo effects. The authors speculated that if patients did not feel particularly well at baseline, they were much more likely to express their symptoms as a side effect (de la Cruz et al., 2010). Physicians may also ameliorate the collection of reports of non-specific side effects to active medications by identifying patients who are most at risk of developing non-specific AEs in the recruitment stage. For example, patients with a tendency to somatization and/or who have significant mood changes are at greater risk of developing non-specific side effects. The use of a specific scale to quantify both of these characteristics in patients with dementia may be helpful in the recruitment stage. It would be important to compare these in the two groups being analyzed (the active medication group versus the placebo group) to describe possible differences if any, in these important psychological aspects, using statistical methods to control bias. 


\section{Conclusions}

This is the first study to show that $\mathrm{AD}$ patients may be at greater risk of developing AEs compared with MCI patients in clinical trials on anti-dementia treatment. This seems to be related to a greater presence of somatic comorbidity which predisposes them to express emotional distress as physical symptoms and/or to $\mathrm{AD}$ patients being frailer and therefore more susceptible to AEs. Here we have suggested that the phenomena that we observed in the $\mathrm{AD}$ population may be interpreted in terms of the nocebo effect. These findings have important implications for both treatment adherence and prognosis, and they indicate a need for a new procedure when conducting RCTs.

\section{Conflict of interest}

None.

\section{Description of authors' roles}

Martina Amanzio designed the review, analyzed the literature search, participated in recording and grouping the AEs, and in writing the paper. Lene Vase analyzed the literature search, participated in the recording and grouping of the AEs, conducted the statistical analyses, and participated in writing the paper. Fabrizio Benedetti supervised the recording and grouping of the AEs and participated in writing the paper.

\section{Acknowledgments}

This study is dedicated to the memory of Dr Luca Latini Corazzini (1972-2009). The authors would like to thank Kathrine Næsted Nørskov for technical assistance. The project was partially funded by the EU-EPIA joint Innovative Medicines Initiative as part of the IMI European Project, grant agreement no. 115007 .

\section{References}

*An asterisk indicates studies that were included in the analysis.

Amanzio, M. (2011). Do we need a new procedure for the assessment of adverse events in anti-migraine clinical trials? Recent Patents on CNS Drug Discovery, 6, 41-47.

Amanzio, M., Latini Corazzini, L., Vase, L. and Benedetti, F. (2009). A systematic review of adverse events in placebo groups of anti-migraine clinical trials. Pain, 146, 261-269.

Amanzio, M. et al. (2011). Unawareness of deficits in Alzheimer's disease: role of the cingulate cortex. Brain, 134, 1061-1076.
Andrykowski, M. and Redd, W. (1987). Longitudinal analysis of the development of anticipatory nausea. Fournal of Consulting E् Clinical Psychology, 55, 36-41.

Benedetti, F. and Amanzio, M. (1997). The neurobiology of placebo analgesia: from endogenous opioids to cholecystokinin. Progress in Neurobiology, 52, 109-125.

Benedetti, F., Lanotte, M., Lopiano, L. and Colloca, L. (2007). When words are painful: unraveling the mechanisms of the nocebo effect. Neuroscience, 147, 260-271.

*Black, S. E. et al. (2007). Donepezil preserves cognition and global function in patients with severe Alzheimer disease. Neurology, 69, 459-469.

*Burns, A. et al. (1999). The effects of donepezil in Alzheimer's disease - results from a multinational trial. Dementia E Geriatric Cognitive Disorders, 10, 237-244.

Cepeda, M. S., Farrar, J. T., Baumgarten, M., Boston, R., Carr, D. B. and Strom, B. L. (2003). Side effects of opioids during short-term administration: effect of age, gender and race. Clinical Pharmacology $\mathcal{E}$ Therapeutics, 74, 102-112.

Davis, C., Ralevski, E., Kennedy, S. H. and Neitzert, C. S. (1995). The role of personality factors in the reporting of side effect complaints to moclobemide and placebo. Fournal of Clinical Psychopharmacology, 15, 347-352.

de la Cruz, M., Hui, D., Parsons, H. A. and Bruera, E. (2010). Placebo and nocebo effects in randomized double-blind clinical trials of agents for the therapy for fatigue in patients with advanced cancer. Cancer, 116, 766-774.

*Doody, R. S. et al. (2009). Donepezil treatment of patients with MCI: a 48-week randomized, placebo-controlled trial. Neurology, 72, 1555-1561.

Doody, R. S. et al. (2010). Safety and tolerability of donepezil in mild cognitive impairment: open-label extension study. American fournal of Alzheimers Disease $\mathbb{E}$ Other Dementias, 25, 155-159.

Doraiswamy, M., Leon, J., Cummings, J. L., Martin, D. and Neumann, PJ. (2002). Prevalence and impact of medical comorbidity in Alzheimer's disease. The fournals of Gerontology Series A, Biological Sciences and Medical Sciences, 57, 73-77.

*Feldman, H., Gauthier, S., Hecker, J., Vellas, B., Subbiah, P. and Whalen, E. (2001). Donepezil MSAD study investigators group: a 24-week, randomized, double-blind study of donepezil in moderate to severe Alzheimer's disease. Neurology, 57, 613-620.

Ferguson, J. M. (1993). Alprazolam XR: patient acceptability, safety, and tolerability. Psychiatric Annals, 23, 20-26.

Folstein, M. F., Folstein, S. E. and McHugh, P. R. (1975). "Mini-mental state": a practical method for grading the cognitive state of patients for the clinician. Fournal of Psychiatric Research, 12, 189-198.

Fried, L. P. et $\boldsymbol{a l}$. (2001). Cardiovascular health study collaborative research group. Frailty in older adults: evidence for a phenotype. Fournals of Gerontology Series A, Biological Sciences and Medical Sciences, 56, M146-M156.

Harrington, A. (ed.) (1997) The Placebo Effect: An Interdisciplinary Exploration. Cambridge, MA: Harvard University Press. 
Hilmer, S. N., McLachlan, A. J. and Le Couteur, D. G. (2007). Clinical pharmacology in the geriatric patient. Fundamental Clinical Neuropharmacology, 21, 217-230.

*Homma, A. et al. (2000). Clinical efficacy and safety of donepezil on cognitive and global function in patients with Alzheimer's disease: a 24-week, multicenter, double-blind, placebo-controlled study in Japan. E2020 study group. Dementia and Geriatric Cognitive Disorders, 11, 299-313.

*Homma, A. et al. (2008). Donepezil treatment of patients with severe Alzheimer's disease in a Japanese population: results from a 24-week, double-blind, placebo-controlled, randomized trial. Dementia and Geriatric Cognitive Disorders, 25, 399-407.

${ }^{*}$ Howard, R. J. et al. (2007). CALM-AD trial group. Donepezil for the treatment of agitation in Alzheimer's disease. New England Fournal of Medicine, 357, 1382-1392.

Jadad, A. R. et al. (1996). Assessing the quality of reports of randomized clinical trials: is blinding necessary? Controlled Clinical Trials, 17, 1-12.

*Johannsen, P. et al. (2006). Assessing therapeutic efficacy in a progressive disease: a study of donepezil in Alzheimer's disease. CNS Drugs, 20, 311-325.

*Mazza, M., Capuano, A., Bria, P. and Mazza, S. (2006). Ginkgo biloba and donepezil: a comparison in the treatment of Alzheimer's dementia in a randomized placebo-controlled double-blind study. European fournal of Neurology, 13, 981-985.

McLean, A. J. and Le Couteur, D. G. (2004). Ageing biology and geriatric clinical pharmacology. Pharmacological Reviews, 56, 163-184.

*Mohs, R. C. et al. (2001). "312” study group. A 1-year, placebo-controlled preservation of function survival study of donepezil in AD patients. Neurology, 57, 481-488.

*Petersen, R. C. et al. (2005). Alzheimer's Disease Cooperative Study Group. Vitamin E and donepezil for the treatment of mild cognitive impairment. New England Fournal of Medicine, 352, 2379-2388.

Raschetti, R., Albanese, E., Vanacore, N. and Maggini, M. (2007). Cholinesterase inhibitors in mild cognitive impairment: a systematic review of randomized trials. PLoS Medicine, 4, 1818-1828.

Rief, W. et al. (2009). Differences in adverse effect reporting in placebo groups in SSRI and tricyclic antidepressant trials: a systematic review and meta-analysis. Drug Safety, 32, 1041-1056.

${ }^{*}$ Rogers, S. L. and Friedhoff, L.T. (1996). The efficacy and safety of donepezil in patients with Alzheimer's disease: results of a US multicentre, randomized, double-blind, placebo-controlled trial. The Donepezil Study Group. Dementia, 7, 293-303.

${ }^{*}$ Rogers, S. L., Doody, R. S., Mohs, R. C. and Friedhoff, L. T. (1998a). Donepezil improves cognition and global function in Alzheimer disease: a 15-week, double-blind, placebo-controlled study. Donepezil Study Group. Archives of Internal Medicine, 158, 1021-1031.

${ }^{*}$ Rogers, S. L., Farlow, M. R., Doody, R. S., Mohs, R. and Friedhoff, LT. (1998b). A 24-week, double-blind, placebo-controlled trial of donepezil in patients with Alzheimer's disease. Donepezil Study Group. Neurology, 50, 136-45.

*Salloway, S. et al. (2004). Donepezil 401 study group. Efficacy of donepezil in mild cognitive impairment: a randomized placebo-controlled trial. Neurology, 63, 651-657.

*Seltzer, B. et al. (2004). Efficacy of donepezil in early-stage Alzheimer disease: a randomized placebo-controlled trial. Archives of Neurology, 61, 1852-1856.

Standaert, D. G. and Young, A. B. (1996). Treatment of central nervous system degenerative disorders. In J. G. Hardman and L. E. Limbird (eds.), Goodman E Gilman's The Pharmacological Basis of Therapeutics (pp. 503-519). New York: Mc Graw-Hill.

*Tariot, P. N. et al. (2001). A randomized, double-blind, placebo-controlled study of the efficacy and safety of donepezil in patients with Alzheimer's disease in the nursing home setting. Fournal of the American Geriatrics Society, 49, 1590-1599.

Waldemar, G. et al. (2007). Recommendations for the diagnosis and management of Alzheimer's disease and other disorders associated with dementia: EFNS guidelines. European fournal of Neurology, 14, e1-e26.

Walston, J. et al. (2006). Research agenda for frailty in older adults: toward a better understanding of physiology and etiology. Summary from the American Geriatrics Society/National Institute on Aging Research Conference on Frailty in Older Adults. Fournal of the American Geriatrics Society, 54, 991-1001.

*Winblad, B. et al. (2001). Donepezil Nordic study group. A 1-year, randomized, placebo-controlled study of donepezil in patients with mild to moderate AD. Neurology, 57, 489-495.

*Winblad, B. et al. (2006). Severe Alzheimer's disease study group. Donepezil in patients with severe Alzheimer's disease: double-blind, parallel-group, placebo-controlled study. Lancet, 367, 1057-1065.

Wolf, S. and Pinsky, R. M. (1954). Effects of placebo administration and occurrence of toxic reactions. $\mathcal{F} A M A$, 155, 339-341. 